BACKGROUND: It has recently been shown that soluble interleukin-6 receptor (sIL-6R) alone or complexed with interleukin (IL)-6, besides their regulatory role in a wide variety of both normal and abnormal biologic reactions mediated by IL-6, could be an effective stimulator of the cell function.

Aims: The key question of the present study is whether the sIL-6R $\alpha$ or sIL-6R with IL-6 released by polymorphonuclear leukocytes (PMN) can influence cytokine secretion such as tumor necrosis factor- $\alpha$ $\left(\right.$ TNF- $\left._{\alpha}\right)$ by peripheral blood mononuclear cells (PBMC), which together with PMN develop the inflammatory and immune response of a host.

Methods: Cells were isolated from heparinized whole blood of healthy persons. The PMN were cultured for $1 \mathrm{~h}$ at $37^{\circ} \mathrm{C}$ in $5 \% \mathrm{CO}_{2}$. After incubation, the culture supernatant of PMN was removed and was added to PBMC. The PBMC were cultured for $1 \mathrm{~h}$ at $37^{\circ} \mathrm{C}$ in the same conditions. In the culture supernatants and lysates of PMN, we examined the concentrations of sIL-6R by enzyme-linked immunosorbent assay (ELISA). TNF- $\alpha$ was measured at both protein and mRNA levels. Protein levels were determined by ELISA. To examine TNF- $\alpha$ mRNA expression, we isolated mRNA from PBMC after culture, using TRIZOI Reagent. The quantity of mRNA TNF- $\alpha$ was determined by the Quantikine mRNA assay.

Results and conclusion: The results obtained revealed that sIL-6R with IL-6 secreted by PMN may play a regulatory role in the immune response by modulating the TNF- $\alpha$ expression and its production by PBMC. This may have a significant influence on an early phase of the inflammation and other reactions mediated by TNF- $\alpha$.

Key words: Polymorphonuclear cells, Peripheral blood mononuclear cells, Interleukin-6, Soluble interleukin-6 receptor, Tumor necrosis factor

\section{Effect of soluble interleukin-6 receptor $\alpha$ and interleukin-6 secreted by polymorphonuclear leukocytes on tumor necrosis factor- $\alpha$ expression and its production by peripheral blood mononuclear cells}

\section{E. Jablonska}

Department of Immunology, Medical Academy of Bialystok, Washington 15A, 15-274 Bialystok, Poland

\author{
${ }^{\mathrm{CA}}$ Corresponding Author \\ Fax: +48 85424907 \\ E-mail: ewaj@amb.edu.pl
}

\section{Introduction}

Polymorphonuclear leukocytes (PMN), abundant cells of an early phase of inflammation, have ability to release a wide range of cytokines and their soluble receptors, which influence the host's non-specific and specific immune response. ${ }^{1}$ Interleukin (IL)- 6 and its soluble receptors, soluble interleukin- 6 receptor $\alpha$ $\left(\right.$ sIL-6R $R_{\alpha}$ ) and sgp130, among the known soluble modulators secreted by PMN, appear to be important for a host's response. ${ }^{2,3}$

IL-6 plays a key role in inducing and maintaining inflammatory responses. ${ }^{3}$ On the contrary, IL-6 acts also as an anti-inflammatory mediator through downregulation of pro-inflammatory cytokines, such as IL$1 \beta$ and $\mathrm{TNF}_{-} \alpha$, and simultaneous induction of the IL-1 receptor antagonist and the soluble p55 TNF- $\alpha$ receptor expression. ${ }^{4}$
The receptor complex mediating the biological activities of IL-6 consists of two membrane-bound proteins, an $80-\mathrm{kDa}$ ligand binding subunit (IL-6R) and a gp 130-kDa signal-transducing subunit. ${ }^{5}$ Both the IL$6 \mathrm{R}$ and gp130 receptors exist in a soluble form.

The soluble IL- $6 R_{\alpha}$, which lacks the transmembrane and cytoplasmic parts, is generated through proteolytic shedding of a membrane-anchored protein (PC-sIL-6R isoform) or released as a product of alternative mRNA splicing (DS-sIL-6R isoform). ${ }^{2,6}$

In contrast to the most soluble cytokine receptors, sIL-6R exerts agonistic activity when complexed with its ligand. It is known that sIL-6R might act as a regulator in a wide variety of both normal and abnormal biologic reactions. The complex sIL-6R/IL-6 is capable of activating cells through interaction with membrane-bound gp130. By means of this mechanism, sIL-6R might render IL-6-responsive cells, which 
by themselves cannot bind IL-6. Furthermore, sIL-6R is a carrier protein for its IL- 6 ligand, prolonging the plasma half-life of IL-6. ${ }^{5,6}$

It has recently been shown that soluble IL-6 receptor alone could be an effective stimulator of the cell function. ${ }^{7,8}$ Modur et al. demonstrated that PMN exposed to fMPL shed IL-6R in sufficient quantities to activate the inflammatory response of endothelial cells. $^{7}$

The key question of the present study is whether the sIL- $6 \mathrm{R}_{\alpha}$ released by PMN can also influence the cytokine secretion by peripheral blood mononuclear cells (PBMC), which together with neutrophils develop the inflammatory and immune response of a host. Since IL- 6 and TNF- $\alpha$ co-stimulate each other's release and IL- 6 is one of the most efficient modulators of $\mathrm{TNF}_{-} \alpha$ production, we examined the effect of sIL-6R and sIL-6R with IL-6 secreted by PMN on $\mathrm{TNF}_{-} \alpha$ expression and its production by PBMC. Our studies of the biological function of sIL-6R and IL-6 secreted by PMN may provide new data on the early phase of inflammation and immune response mediated by these cells.

\section{Material and methods}

Cells were isolated from heparinized $(10 \mathrm{U} / \mathrm{ml})$ whole blood of 12 healthy persons by Gradisol $\mathrm{G}$ gradient $(1.115 \mathrm{~g} / \mathrm{ml})$. This method enables the simultaneous separation of two highly purified leukocyte fractions: PBMC containing 95\% lymphocytes, and polymorphonuclear cells cont. 94\% PMN. The purity of isolated PMN was determined by May-Grunewald-Giemsa staining. The cells were washed three times with RPMI 1640 medium (Gibco, Paisley, Scotland) and suspended in the culture medium to provide $5 \times 10^{6}$ cells $/ \mathrm{cm}^{3}$. The culture medium consisted of RPMI 1640 medium supplemented with 10\% autologous serum (Pro Animal, Wroclaw, Poland), $100 \mathrm{U} / \mathrm{ml}$ of penicillin and $50 \mathrm{ng} / \mathrm{ml}$ of streptomycin.

The PMN were cultured in flat-bottomed, 96-well plates (Falcon, Franklin Lakes, USA) for $1 \mathrm{~h}$ at $37^{\circ} \mathrm{C}$ in a humidified incubator with $5 \% \mathrm{CO}_{2} \quad$ (Nuaire $^{\mathrm{TM}}$, Minnesota, USA). After $1 \mathrm{~h}$ incubation, the culture supernatant of PMN was removed and added to PBMC $\left(5 \times 10^{6}\right.$ cells $\left./ \mathrm{cm}^{3}\right)$. The viability of $\mathrm{PMN}$ was $>92 \%$ as determined by trypan blue exclusion. The PBMC were cultured for $1 \mathrm{~h}$ at $37^{\circ} \mathrm{C}$ in the same conditions. PBMC with the culture supernatants of PMN and monoclonal antibodies anti-sIL-6R $(20 \mathrm{ng} / \mathrm{ml})$ and anti-IL-6 (20 $\mathrm{ng} / \mathrm{ml})$ were the negative control, whereas PBMC with recombinant human (rh) sIL-6R (Endogen, Woburn, USA) and rhIL-6 (R\&D, Minneapolis, USA) were the positive control.

In the culture supernatants and lysates of PMN, we examined the concentrations of sIL-6R by the human sIL-6R enzyme-linked immunosorbent assay (ELISA) (Biosource, Nivelles, Belgium).
TNF- $\alpha$ was measured at both protein and mRNA levels. Protein levels were determined by the Human TNF- $\alpha$ ELISA (Biosource). To examine TNF- $\alpha$ mRNA expression, we isolated mRNA from PBMC after culture, using TRIZOL Reagent (Gibco), according to the manufacturer's instruction. The quantity of mRNA TNF- $\alpha$ was determined by the Quantikine mRNA assay (Base Kit, Quantikine mRNA Probes and Calibrator Kit - Human TNF- $\alpha$; R\&D Systems).

The concentration of RNA samples was determined at $A_{260}$ and $A_{280}$ using absorbance values in the linear range of the spectrophotometer. The $A_{260} / A_{280}$ ratio for RNA samples was 1.8-1.9.

\section{Results}

TNF- $_{\alpha}$ mRNA in PBMC

In the PBMC, after the culture with PMN supernatants, we found a lower expression of TNF- $\alpha$ mRNA than in PBMC cultured without supernatants from PMN (Fig. 1). Anti-sIL-6R alone and anti-sIL-6R with anti-IL-6 monoclonal antibodies were used to confirm whether the actions of PMN supernatant were generated by these proteins. In the presence of anti-sIL6R antibodies alone, the expression of TNF- $\alpha$ mRNA in PBMC cultured with PMN supernatants was not changed. In contrast, in the presence of anti-sIL-6R with anti-IL-6 monoclonal antibody, the expression of TNF- $_{\alpha}$ mRNA in PBMC was higher than in the PBMC cultured without PMN supernatants (Fig. 1A).

We examined $\mathrm{TNF}_{\alpha} \alpha$ expression in PBMC in the presence of rhsIL-6R alone and in the presence of rhsIL-6R with rhIL-6 to confirm the observations just mentioned. A similar expression of $\mathrm{TNF}_{-} \alpha$ mRNA was found in PBMC cultured without PMN supernatant and PBMC cultured with rhsIL-6R alone. In the presence of rhsIL-6R and rhIL-6, the expression of TNF- $\alpha$ mRNA in PBMC was lower than in PBMC cultured without PMN supernatant.

\section{TNF- $_{\alpha}$ concentrations in the culture} supernatants of PBMC

TNF- $_{\alpha}$ concentrations in the culture supernatants of PBMC cultured in the presence of PMN supernatants were lower than those in the supernatants of PBMC cultured without PMN supernatants (Fig. 1B).

The presence of anti-sIL-6R antibody in the culture of PBMC with PMN supernatants induced the same level of $\mathrm{TNF}_{-} \alpha$ secretion as in the culture of PBMC alone. In the presence of anti-sIL-6R with anti-IL- 6 , the concentration of $\mathrm{TNF}_{-} \alpha$ in the PBMC supernatant was higher than in the culture supernatant of PBMC cultured with PMN supernatant. We stimulated PBMC with rhsIL-6R alone and rhsIL-6R with rhIL-6 to confirm the effect of these mediators secreted by 
$\mathbf{A}$

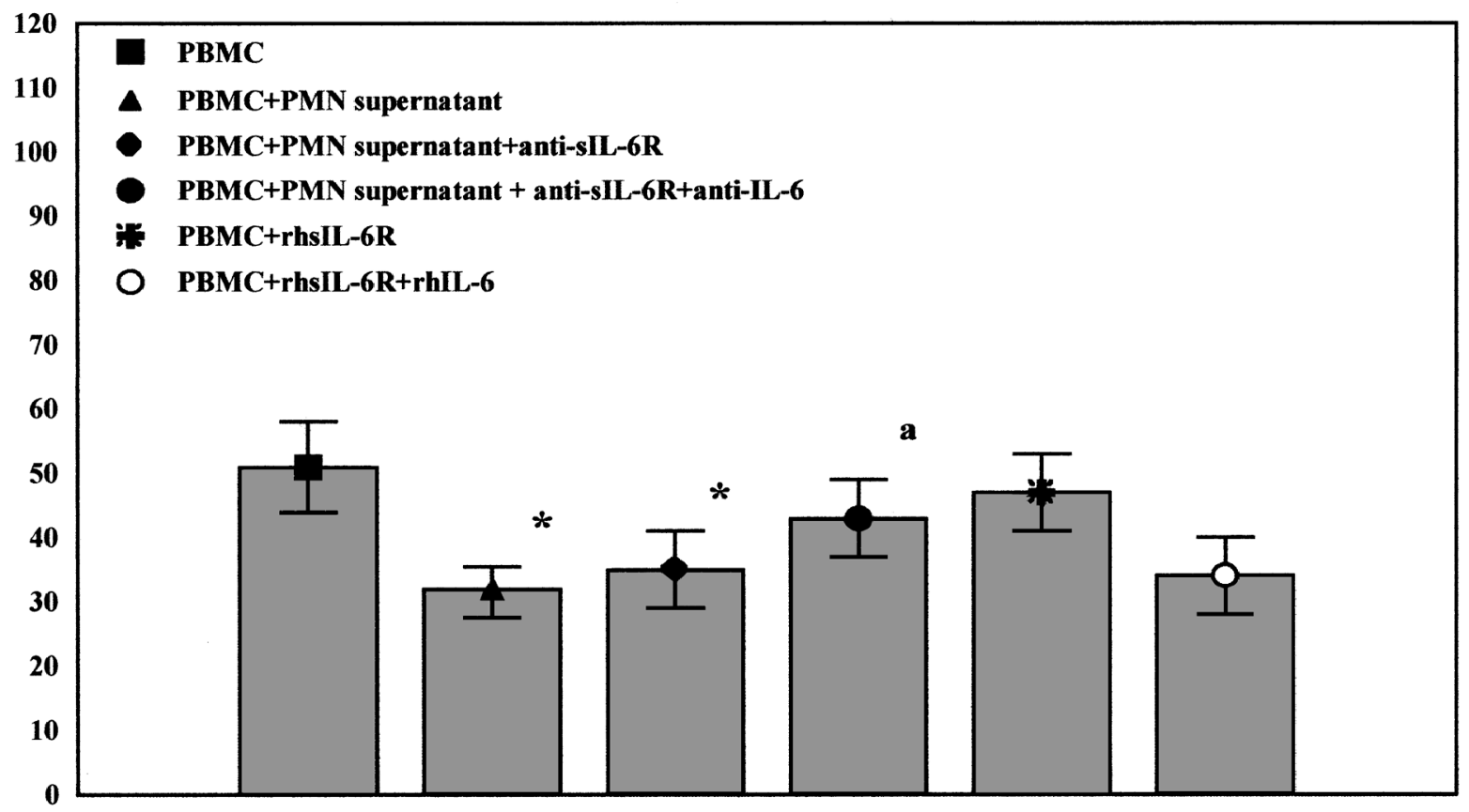

B

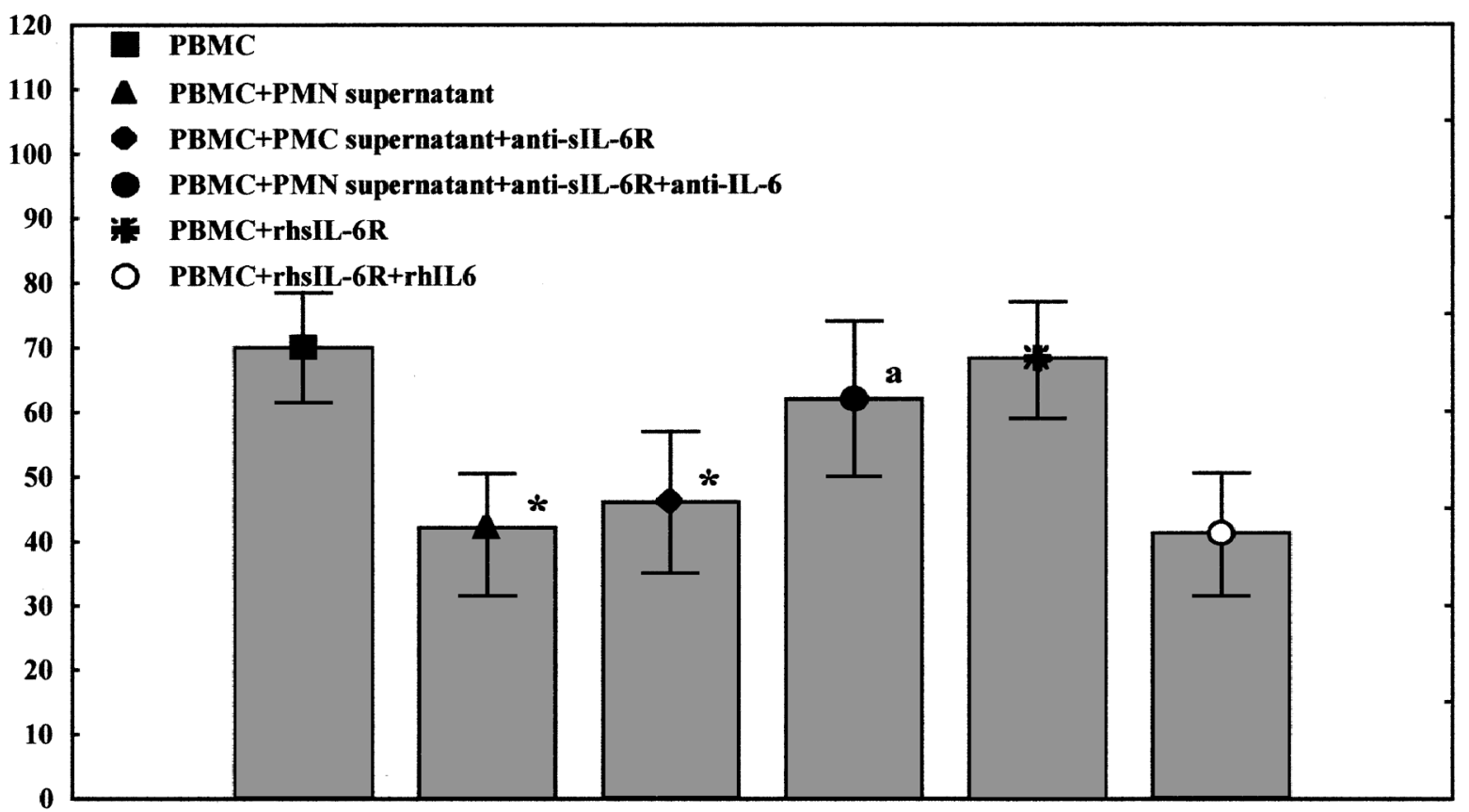

FIG. 1. TNF- ${ }_{\alpha}$ expression and its production by unstimulated and stimulated PBMC. (A) TNF- $\alpha$ mRA expression ( ${ }_{\mu} \mathrm{mol} / \mathrm{ml}$ ) and (B) TNF- $\alpha$ concentration in the culture supernatant of PBMC $(\mathrm{pg} / \mathrm{ml})$. ${ }^{*}$ Statistical difference with unstimulated PBMC $(p<0.05)$, ${ }^{a}$ statistical difference with PBMC cultured with PMN supernatant and anti-sIL-6R and anti-IL-6 $(p<0.05)$.

PMN on TNF- $\alpha$ production by PBMC. The concentrations of TNF- $\alpha$ in the culture supernatants of PBMC with rhsIL-6R were no changed. The secretion ofTNF$\alpha$ by PBMC in the presence of rhsIL-6R with rhIL-6 was lower than secretion by PBMC without PMN supernatant.

\section{Discussion}

Soluble forms of receptors are known to be more general phenomena and may play a wider physiological role in the regulation of the immune system than recognized previously. ${ }^{2}$ 
The results obtained indicate that sIL-6R with IL-6 secreted by PMN belong to the factors responsible for the TNF- $\alpha$ expression and its production by autologous PBMC. Joyce et al. showed that induction of PMN can lead to synthesis of other cytokines, such as IL-1, granulocyte-macrophage colony-stimulating factor or $\mathrm{TNF}_{-} \alpha$, which have also been demonstrated to induce the TNF- $\alpha$ production by mononuclear cells. ${ }^{9}$

There are different data referring to the biological activity of sIL-6R alone or complexed with IL-6. Modur et al. found that sIL-6R did not require the addition of exogenous IL- 6 to the surface expression of E-selectin, intracellular adhesion molecule-1, vascular cell adhesion molecule- 1 and the synthesis of IL8 and IL- 6 by these cells. ${ }^{7}$ However, our observations suggest that sIL-6R alone does not exert an effect on TNF- $_{\alpha}$ expression in PBMC.

The observations referring to the sIL-6R and IL-6 function seem to be similar to the other's results and appear to confirm the inhibitory inter-relations between IL- 6 and $\mathrm{TNF} \alpha \cdot{ }^{8,10}$ Schindler et al. reported that IL-6 suppressed TNF- $\alpha$ production by human blood mononuclear cells. ${ }^{11}$ Oh et al. demonstrated that the IL-6/sIL-6R complex inhibited TNF- $\alpha$-induced vascular cell adhesion molecule-1 expression, whereas it did not affect intracellular cell adhesion molecule-1 expression. ${ }^{8}$

There are available data indicating that IL- 6 and $\mathrm{TNF}^{-} \alpha$ can be modulated by their soluble receptors and vice versa. ${ }^{4,6}$ For example, IL- 6 has been found as a stimulator of soluble TNFRp55 receptor secretion. This receptor retains the capacity of binding TNF and may directly neutralize or modulate TNF activity as well as possibly indirectly influencing the IL- 6 function. ${ }^{10}$ Igaz et al. described the inhibiting action of the sIL-6R/IL- 6 on the TNF- $\alpha$ secretion but not on the mRNA TNF- $\alpha$ expression by Jurkat cells. They also found that sIL-6R alone did not influence the TNF- $\alpha$ production by these cells. $^{12}$

However, little is known about the biological role of a second IL-6 soluble receptor, sgp130, also secreted by PMN and PBMC. Soluble gp130 has been shown to bind the bio-active IL-6/IL-6R complex and to inhibit its function. ${ }^{6,13}$ In our earlier studies, we demonstrated the ability of PMN to release sgp130 at a 10-fold higher level than sIL-6R. ${ }^{14}$ The presence of the relatively large amount of sgp130 in the culture medium may affect differently the biological function of sIL-6R. The presence of sgp130, inhibiting IL-6 activity in the culture supernatants of PMN in a paracrine fashion as well as of PBMC in an autocrine fashion, may be directly responsible for the modulation of TNF- $\alpha$ expression and its production by PBMC. Moreover, the recent data of Mueller-Neuwen et al. indicate that sIL-6R enhances the antagonistic effect of sgp130 and that this effect is more pronounced at higher concentrations of IL-6, sIL-6R and sgp130. ${ }^{15}$
Furthermore, the role of both isoforms of sIL-6R produced by PMN in the immune response should be considered when determining the properties of sIL6R. It is known that the PC-sIL-6R and DS-sIL-6R isoforms may independently contribute to the action of this receptor. ${ }^{6}$ In the present examinations, we derived the sIL-6R from the conditioned medium of PMN within $60 \mathrm{~min}$. The sIL-6R obtained in these examinations may be the PC-sIL-6R isoform that can be detected within the first 30-120 min of stimulation. $^{6}$

In conclusion, the results obtained revealed that sIL-6R with IL-6 secreted by human PMN may play a regulatory function in the immune response by modulating the $\mathrm{TNF}_{-} \alpha$ expression and its production by PBMC. Decreased TNF- $\alpha$ expression and its production by sIL-6R and IL-6-stimulated PBMC may have a significant influence on an early phase of the inflammation, mediated by TNF- $\alpha \cdot{ }^{8,10}$ The data presented can form a base for clinical research in the course of infections, in which IL- 6 and TNF- $\alpha$, as well as their soluble receptors, play a key role.

\section{References}

1. Cassatella MA, Gasperini S, Russo MP. Cytokine expression and release by neutrophils. Ann NY Acad Sci 1997; 15: 233-242.

2. Horiuchi S, Koyanagi Y, Zhou Y, et al. Soluble interleukin-6 receptors released from $\mathrm{T}$ cell or granulocyte/macrophage cell lines and human peripheral blood mononuclear cells are generated though an alternative splicing mechanism. Eur J Immunol 1994; 24: 1945-1948.

3. Kishimoto T, Akira S, Narazaki M, Taga T. Interleukin-6 family of cytokines and gp 130. Blood 1995; 86: 1243-1254.

4. Xing Z, Gauldie J, Cox G, Baumann H, Jordana M, Lei X-F, Achong MK. IL-6 Is an antiinflammatory cytokine required for controlling local or systemic acute inflammatory responses. J Clin Invest 1998; 101: 311-320.

5. Müllberg J, Oberthür W, Lottspeich F, et al. The soluble human IL-6 receptor. J Immunol 1994; 152: 4958-4968.

6. Jones SA, Horiuchi S, Topley N, Yamamoto N, Fuller GM. The soluble interleukin 6 receptor: mechanisms of production and implications in disease. FASEB J 2001; 15: 43-58.

7. Modur V, Li Y, Zimmerman GA, Prescott SM, McIntyre TM. Retrograde inflammatory signaling from neutrophils to endothelial cells by soluble interleukin-6 receptor alpha. J Clin Invest 1997; 100: 2752-2756.

8. Oh JW, Van Wagoner NJ, Rose-John S, Benveniste EN. Role of IL-6 and soluble IL-6 receptor in inhibition of VCAM-1 gene expression. J Immunol 1998; 161: 4992-4999.

9. Joyce DA, Steer JH. IL-4, IL-19 and IFN- $\gamma$ have distinct, but interacting, effects on differentiation-induced changes in $\mathrm{TNF}_{-} \alpha$ and TNF receptor release by human monocytes. Cytokine 1996; 8: 49-56.

10. Abe Y, Osuka Y, Nakata T, Kashu Y, Kimura S. The functional role of 55 and $75-\mathrm{kDa}$ tumor necrosis factor receptors in human polymorphonuclear cells in vitro. Cytokine 1995; 7: 39-49.

11. Schindler R, Mancilla J, Endres S, Ghorbani R, Clark SC, Dinarello CA. Correlations and interactions in the production of interleukin-6 (IL-6), IL1 , and tumor necrosis factor (TNF) in human blood mononuclear cells: IL-6 suppresses IL-1 and TNF. Blood 1990; 75: 40-47.

12. Igaz $\mathrm{P}$, Horváth $\mathrm{A}$, Horváth $\mathrm{B}$, et al. Soluble interleukin-6 receptor (sIL-6R) makes IL-6R negative $T$ cell line respond to IL-6; it inhibits TNF production. Immunol Lett 2000; 71: 143-148.

13. Gaillard JP, Mani JC, Liautard J, Klein B, Brochier J. Interleukin-6 receptor signaling. I. Gp80 and gp130 receptor interaction in the absence of interleukin-6. Eur Cytokine Network 1999; 10: 43-48.

14. Jablonska E, Jablonski J. Effect of IL-18 on the release of IL-6 and its soluble receptors - sIL-6R $\alpha$ and sgp130 by human neutrophils. Immunol Invest (2002) 31, 159-161.

15. Müller-Newen G, Küster A, Hemmann U, et al. Soluble IL-6 receptor potentiates the antagonistic activity of soluble gp130 on IL-6 responses. J Immunol 1998; 161: 6347-6355. 


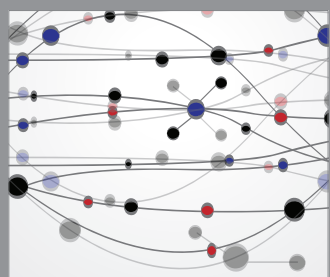

The Scientific World Journal
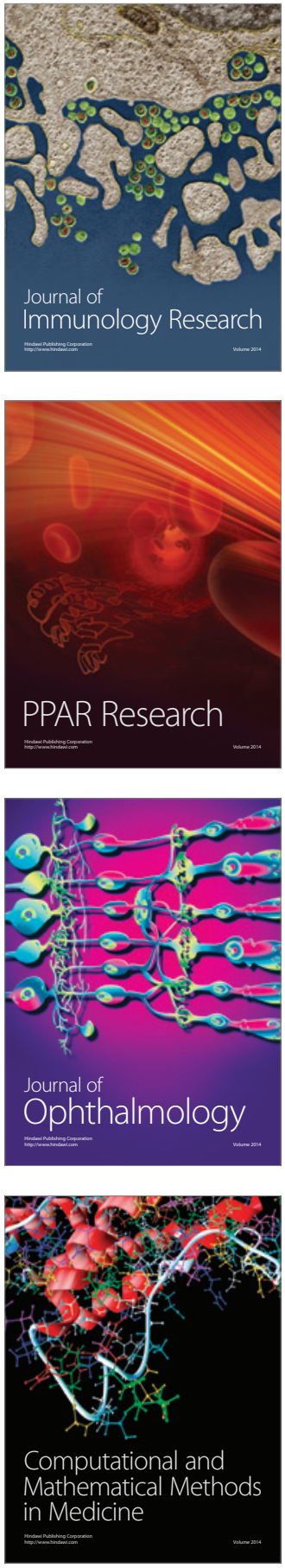

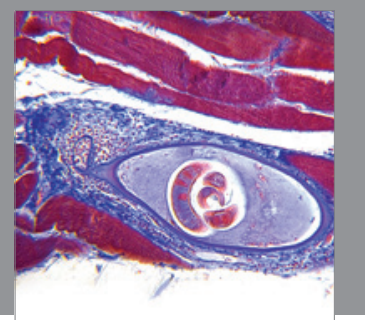

Gastroenterology

Research and Practice
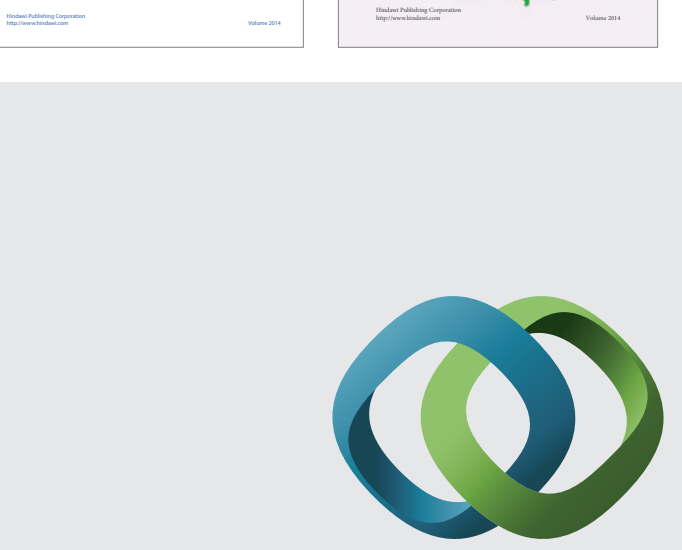

\section{Hindawi}

Submit your manuscripts at

http://www.hindawi.com
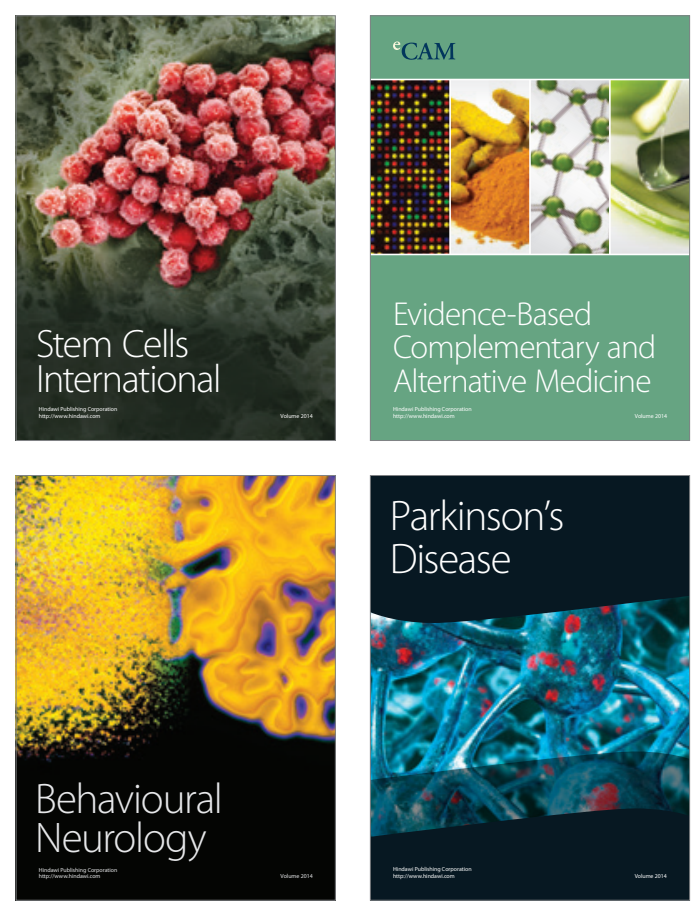

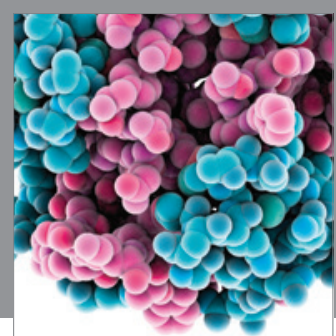

Journal of
Diabetes Research

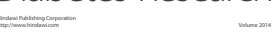

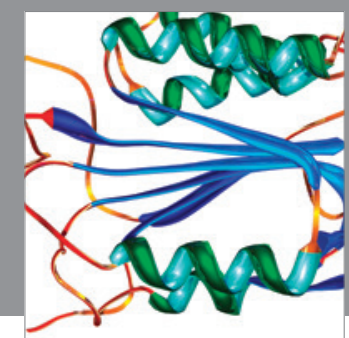

Disease Markers
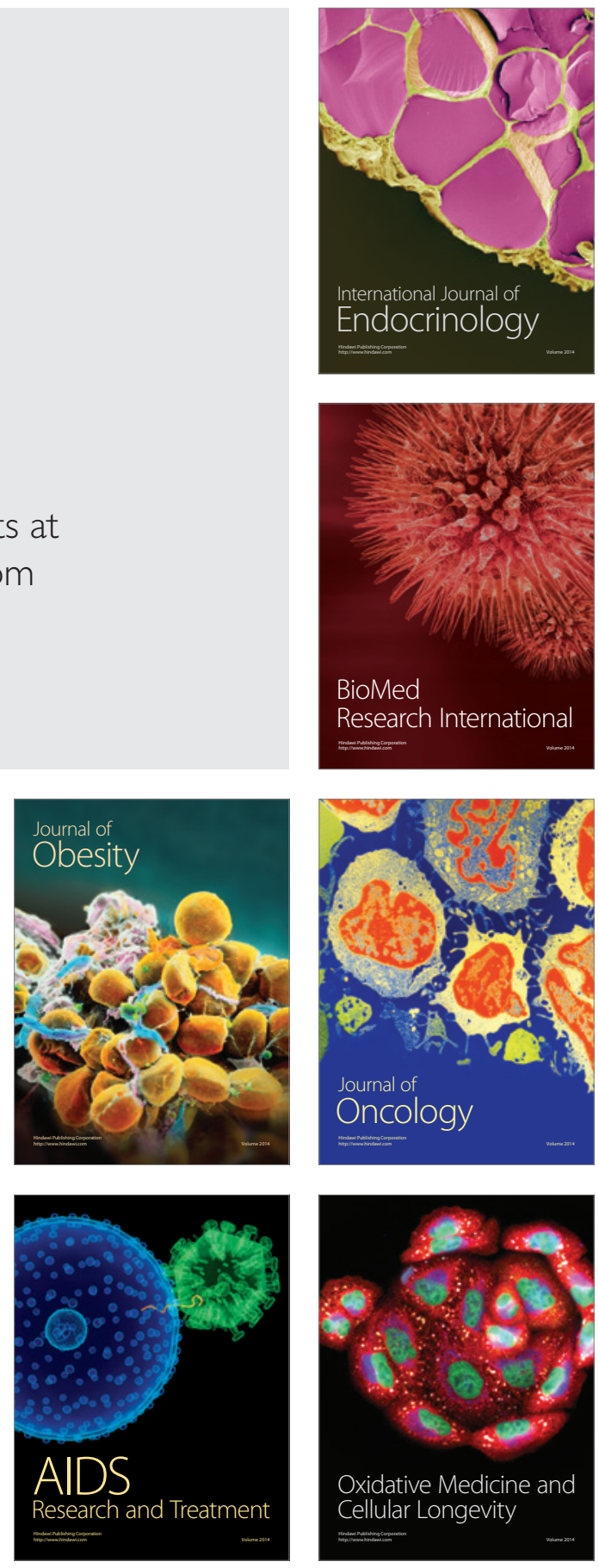